\title{
The Correlation of EMT-related Genes in the Pathogenesis of Non-Small Cell Lung Cancer and Chronic Obstructive Pulmonary Disease
}

\section{Lian-Gang Li ( $\square$ liliangang@aliyun.com )}

The Third People's Hospital of Yuhang District https://orcid.org/0000-0003-4739-7925

\section{Hangqierang Cai}

Qinghai University Affiliate Hospital

\section{Xiao-Bo Zhou}

Qinghai University Affiliated Hospital

\section{Lan Zhang}

Qinghai University Affiliated Hospital

\section{Tai Jiu}

Qinghai University Affiliated Hpspital

\section{Zhi-Bo Liu}

Qinghai University Affiliated Hospital

\section{Fang Hui}

Qinghai University Affiliated Hospital

Ya-Jun Tuo

Qinghai Provincial People's Hospital

Jun Jiang

Qinghai University Affiliate Hospital

\section{Research}

Keywords: Epithelial-mesenchymal transformation, non-small cell lung cancer, chronic obstructive pulmonary disease, ITGB1, VIM, KRT14, MMP-9, Notch1

Posted Date: October 13th, 2021

DOI: https://doi.org/10.21203/rs.3.rs-915753/v1

License: (c) (i) This work is licensed under a Creative Commons Attribution 4.0 International License.

Read Full License 


\section{Abstract}

Purpose: To analyze the expression of epithelial-mesenchymal transformation (EMT)-related genes in non-small cell lung cancer (NSCLC) and chronic obstructive pulmonary disease (COPD).

Methods: Bronchoalveolar lavage fluid samples were collected from patients diagnosed with NSCLC, COPD, and COPD complicated with NSCLC. RNA from cells was extracted with Trizol reagent. Differential genes were screened and validated using the PCR Array and qRT-PCR, respectively.

Results: The expressions of ITGB1, VIM, and MMP-9 were higher in the NSCLC group compared to the COPD group $(P<0.05)$. Moreover, the expressions of ITGB1, VIM, MMP-9, and Notch1 were higher, and the expression of KRT14 was lower in the complicated group compared to the NSCLC group $(P<0.05)$.In the NSCLC subgroup, MMP-9 and Notch1 were highly expressed in the lung adenocarcinoma group $(P<0.05)$ compared to the squamous cell lung carcinoma group.

Conclusions: The upregulation of ITGB1, VIM, MMP-9, and Notch1 and downregulation of KRT14 might be closely related to the occurrence and development of NSCLC, mainly lung adenocarcinoma.

\section{Background}

Lung cancer is a type of tumor with high morbidity and mortality rates, usually originating from bronchial mucosa epithelium ${ }^{[1,2]}$. Clinically, $80-85 \%$ of lung cancer cases are diagnosed with non-small cell lung cancer (NSCLC) consisting of adenocarcinoma, squamous cell carcinoma, and large cell lung carcinoma $^{[3]}$. In addition, chronic obstructive pulmonary disease (COPD) refers to another group of chronic lung diseases that affect the structure and function of large, medium, and small airways and alveoli, causing a continuous and irreversible airflow restriction ${ }^{[4]}$. About 600 million people worldwide are currently suffering from COPD, with a roughly $10 \%$ morbidity and a death toll of around 3 million per year ${ }^{[5,6]}$.

NSCLC and COPD are two independent diseases with entirely different prognoses. Smoking, the same context of lung cells and tissues, high-incidence populations, hereditary factors, and chronic respiratory tract inflammation are the common predisposing factors in both NSCLC and COPD ${ }^{[7,8]}$. Moreover, an increasing number of studies have suggested that epithelial-mesenchymal transition (EMT) has an essential role in the progression of NSCLC and COPD ${ }^{[9,10]}$. EMT is a process during which epithelial cells lose their inherent polarity and viscosity and gain infiltrative, migratory, and invasive behavior transforming into mesenchymal cells ${ }^{[11]}$. EMT participates in the growth and development of tissues, embryos, and organs and the repair process after injury. It also has an important role in tumor cells' occurrence, invasion, and metastasis ${ }^{[12]}$.

EMT is regulated by EMT-related genes ${ }^{[13]}$.The fundamental alterations in epithelial cells are caused by genomic and transcriptomic reprogramming ${ }^{[14,16]}$.In lung cancer, these changes have been associated 
with the initiation and metastasis oftumor cells ${ }^{[3]}$. On the other hand, EMT has been associated with airway remodeling and obliteration in COPD ${ }^{[10]}$. Yet, whether COPD and NSCLC are regulated by different or the same EMT activity remains unexplored.

In this study, we investigated the expression of EMT-related genes as an entry point in the lower cell precipitate of bronchoalveolar lavage fluid from patients with NSCLC, COPD, and those with COPD complicated with NSCLC, to roughly reflect the correlation of EMT in the pathogenesis of NSCLC and COPD.

\section{Material And Methods}

\section{Patients and Clinical Samples}

In this study, atotal of 66 centrifuged lower cell precipitate samples were collected from the bronchoalveolar lavage fluid of NSCLC patients (30 samples), COPD patients (28 samples), and NSCLC patients (8 samples) complicated with COPD, who received treatment at Qinghai University Affiliated Hospital and Qinghai Provincial People's Hospital. The inclusion criteria were: 1) the patients diagnosed with NSCLC according to the guidelines of the National Comprehensive Cancer Network (NCCN) and those with COPD according to the guidelines of GOLD;2) no comorbidities that interfered with the test detection, such as tuberculosis, pneumonia, pulmonary fibrosis, and so on;3) patients with NSCLC not receiving treatments(chemotherapy/radiation); 4) those who signed the informed consent.

For the NSCLC and the complication group patients, epithelial cells in the airway were collected from the tumor location. In order to collect the same amount of cells in each group, we used a sorter to extract a relatively small number of epithelial cells from the scattered cells and then focused on processing these cells to ensure that there were enough airway epithelial cells to provide RNA.

The number of samples used for gene chip screening in the PCR Array experiment in the early stage was 12 , with 4 samples [including 3 men and 1 woman, average age $(60.14 \pm 10.72)$ ] from the NSCLC group, the COPD group, and the complication group, respectively. The number of samples used for gene chip validated using quantitative real-time PCR(qRT-PCR) experiment was 54, including 26 samples[including 12 men and 14 women, average age (61.02 \pm 10.46$)$ ] from the NSCLC group, 24 samples [including 17 men and 7 women, average age $(60.04 \pm 10.14)$ ]from the COPD group, and 4 samples [all of men, average age $(67.51 \pm 7.48)$ ] from the complication group. After the centrifugation of all the samples, the cell precipitates were stored in the refrigerator at $-80^{\circ} \mathrm{C}$ until further testing.

\section{Rna Extraction}

Total RNA was extracted from the fresh and frozen samples with the TRIzol method. Briefly, samples were mixed with TRIzol reagent and chloroform and then centrifuged at $4^{\circ} \mathrm{C}$ and $12,000 \mathrm{rpm}$ for $15 \mathrm{~min}$. RNA was purified from the upper/aqueous phase of the TRIzol extract. The concentration and purity of 
RNA were then evaluated with a nucleic acid-protein quantitative instrument, and the integrity of RNA was tested by electrophoresis in $2 \%$ agarose gel.

\section{Screening Of Emt-related Genes}

A cDNA was synthesized from total RNA through reverse transcription according to the PCR procedure (Table S10). EMT-related genes were selected based on Gene chip screening and Bioinformatics analysis. The PCR Array screening protocol is shown in Figure. 1.

PCR reaction solution was compounded according to the requirements of Table S9. Briefly, 25 $\mu$ l of PCR reaction solution was put into each well (96-well PCR reaction plate). Impurities were removed after 1minute centrifugation in a 2000rpm centrifuge, and each well was double-checked.

After gene chip screening, 92 potential EMT-related genes were identified to evaluate gene multi-group expression levels (Table S1). Based on the above gene chip data (Table S2-7, Figure. S1-9), differential gene screening was performed only on genes with the $P$-value $<0.05$ after pairwise comparisons among the three disease groups. Then based on a comprehensive literature review, the function of each gene was retrieved from the gene databases of the National Center for Biotechnology Information (NCBI), and the genes associated with immune inflammation and tumor were selected as candidate genes.

The gene pathway enrichment analysis was performed usingthe Kyoto Encyclopedia of Genes and Genomes (KEGG) to determine the gene-related signaling pathways and select the genes located in the inflammation-related signaling pathway as the candidate genes. The gene co-expression network was drawn using the STRING software to determine the genes located at key nodes. After the above bioinformatics analysis, five genes with significant fold changes, i.e., ITGB1, VIM, KRT14, MMP-9, and Notch1, were finally selected as the candidate validation genes, as shown in Table 1 and Table S8. 
Table 1

PCR Array screening test results

\begin{tabular}{|c|c|c|c|c|c|c|}
\hline Symbol & $\begin{array}{l}\text { FC } \\
\text { (NSCLC } \\
\text { group } \\
\text { vs. } \\
\text { COPD } \\
\text { group) }\end{array}$ & $\begin{array}{l}\text { P-value } \\
\text { (NSCLC } \\
\text { group } \\
\text { vs. } \\
\text { COPD } \\
\text { group) }\end{array}$ & $\begin{array}{l}\text { FC } \\
\text { (Complication } \\
\text { group vs. } \\
\text { COPD group) }\end{array}$ & $\begin{array}{l}P \text {-value } \\
\text { (Complication } \\
\text { group vs. } \\
\text { COPD group) }\end{array}$ & $\begin{array}{l}\text { FC } \\
\text { (Complication } \\
\text { group vs. } \\
\text { NSCLC } \\
\text { group) }\end{array}$ & $\begin{array}{l}P \text {-value } \\
\text { (Complication } \\
\text { group vs. } \\
\text { NSCLC } \\
\text { group) }\end{array}$ \\
\hline ITGB1 & 14.36 & 0.007 & 13.54 & 0.010 & & \\
\hline VIM & 11.58 & 0.023 & 30.27 & 0.029 & & \\
\hline KRT14 & & & -3.14 & 0.013 & -2.36 & 0.022 \\
\hline MMP-9 & & & 11.44 & 0.038 & & \\
\hline Notch1 & & & 3.73 & 0.012 & & \\
\hline
\end{tabular}

\section{Validation of Selected Genes}

We conducted the qRT-PCR experiment by selecting more samples for gene chip validation. Thesampling protocol for the qRT-PCR is shown in Figure. 2.

To validate the selected genes, expression levels were evaluated in a larger series of the NSCLC group (26 samples), the COPD group(24 samples), and the complication group (4 samples). cDNA was synthesized from 300ng of total RNA using TransPlex® Whole Transcriptome Amplification (WTA) Kit (Sigma-

Aldrich $\AA$ ) according to manufacturer's protocol. WTA reaction products were purified using QIAquick PCR Purification Kit, according to manufacturer's protocol. mRNA levels were evaluated using TaqMan® Gene Expression assays for the selected genes.

The qRT-PCR assay was performed in 96-well plates on an Applied Biosystems 7500 Real-time PCR system according to the PCR system(Table S11) and program(Table S12).The qRT-PCR assay was used to quantifythe expression ofITGB1,VIM, KRT14, MMP-9, and Notch1 by amplifying the primers of each gene(Table S13).

\section{NSCLC subgroup study}

NSCLC patients were further divided into two subgroups(Figure. 3): lung adenocarcinoma group and squamous cell lung carcinoma group.

\section{Statistical analysis}


The SPSS19.0 software was used to organize the experimental data and make statistical analyses. The measurement data is represented by the mean \pm standard deviation (mean $\pm S D$ ). If the measurement data conformed to the normal distribution, the ANOVA method was used for the comparison of variables among groups, and the LSD method for pairwise comparisons; t-test was used for the comparison of variables between groups. If there was conformity, the logarithmic conversion was carried out to normalize the data, after which the data were statistically analyzed using the above one-way ANOVA method. The measurement data were described in terms of ratio or constituent ratio, and the Chi-square test was used for the comparison of differences between groups in the measurement data. The inspection level $a=0.05$ indicated statistically significant differences.

\section{Results}

\section{Clinical characteristics data expression}

No statistical differences in gender, age, ethnic group, smoking status, altitude of living area, and complicating disease were found between the 3 groups $(P>0.05)$ (Table 2$)$. 
Table 2

Comparison of clinical characteristics NSCLC group, COPD group and complication group $[\mathrm{n}(\%)]$

\begin{tabular}{|c|c|c|c|c|c|c|}
\hline \multirow[t]{2}{*}{ Item } & & $\begin{array}{l}\text { NSCLC } \\
\text { group }\end{array}$ & $\begin{array}{l}\text { COPD } \\
\text { group }\end{array}$ & $\begin{array}{l}\text { Complication } \\
\text { group }\end{array}$ & \multirow[t]{2}{*}{$\mathrm{X} 2$} & \multirow[t]{2}{*}{$\mathbf{P}$} \\
\hline & & $\mathrm{N}=26$ & $\mathrm{~N}=24$ & $\mathrm{~N}=4$ & & \\
\hline \multirow[t]{2}{*}{ Gender } & M & $12(46.2)$ & $17(70.8)$ & $4(100.0)$ & \multirow[t]{2}{*}{5.460} & \multirow[t]{2}{*}{0.056} \\
\hline & $\mathrm{F}$ & $14(53.8)$ & $7(29.2)$ & $0(0)$ & & \\
\hline \multirow[t]{3}{*}{ Age } & $\leq 44$ & $3(11.5)$ & $1(4.2)$ & $0(0)$ & \multirow[t]{3}{*}{1.845} & \multirow[t]{3}{*}{0.808} \\
\hline & $\begin{array}{l}45- \\
59\end{array}$ & $10(38.5)$ & 8(33.3) & $1(25.0)$ & & \\
\hline & $\geq 60$ & $13(50.0)$ & $15(62.5)$ & $3(75.0)$ & & \\
\hline \multirow{2}{*}{\multicolumn{2}{|c|}{$\begin{array}{l}\text { Han Nationality } \\
\text { Minority Ethnic } \\
\text { Groups }\end{array}$}} & $22(84.6)$ & 16(66.7) & $3(75.0)$ & \multirow[t]{2}{*}{2.342} & \multirow[t]{2}{*}{0.289} \\
\hline & & $4(15.4)$ & 8(33.3) & $1(25.0)$ & & \\
\hline \multirow{2}{*}{\multicolumn{2}{|c|}{$\begin{array}{l}\text { Smoker } \\
\text { Non-smoker }\end{array}$}} & $12(46.2)$ & 17(70.8) & $4(100.0)$ & \multirow[t]{2}{*}{5.460} & \multirow[t]{2}{*}{0.056} \\
\hline & & 14(53.8) & $7(29.2)$ & $0(0)$ & & \\
\hline \multirow{2}{*}{\multicolumn{2}{|c|}{$\begin{array}{l}\text { High Altitude } \\
\text { Plain }\end{array}$}} & $25(96.2)$ & $21(87.5)$ & $4(100.0)$ & \multirow[t]{2}{*}{1.437} & \multirow[t]{2}{*}{0.519} \\
\hline & & $1(3.8)$ & $3(12.5)$ & $0(0)$ & & \\
\hline \multirow{2}{*}{\multicolumn{2}{|c|}{$\begin{array}{l}\text { Complications } \\
\text { No Complications }\end{array}$}} & $20(76.9)$ & $14(58.3)$ & $2(50.0)$ & \multirow[t]{2}{*}{2.654} & \multirow[t]{2}{*}{0.299} \\
\hline & & $6(23.1)$ & $10(41.7)$ & $2(50.0)$ & & \\
\hline \multicolumn{7}{|c|}{$\begin{array}{l}\text { Notes: Smoker consumes at least one fuel-cured tobacco everyday (cannabis and e-cigarette } \\
\text { excluded); complications mainly refer to diabetes, hypertension, coronary heart diseases, and other } \\
\text { types of tumor, with very small quantities.According to international conventions, high altitude refers } \\
\text { to } \geq 1500 \mathrm{~m} \text {. }\end{array}$} \\
\hline
\end{tabular}

\section{Expression of EMT-related genes in NSCLC, COPD, and COPD complicated with NSCLC}

The results of the qRT-PCR validation experiment showed that ITGB1, VIM, KRT14, MMP-9, and Notch1 were differentially expressed among patients with COPD, NSCLC, and complicated groups (Table 3 and Figure. 4). The expression of ITGB1, VIM, and MMP-9 was significantly higher in the NSCLC group compared to the COPD group ( $<<0.05)$. Moreover, the expression of ITGB1, VIM, MMP-9, and Notch1 was higher, while the expression of KRT14 was lower in the complicated group than in the NSCLC group $(\mathrm{P}<$ 0.05). We concluded that upregulation of ITGB1, VIM, MMP-9, and Notch1 and downregulation of KRT14 couldbe related to the occurrence and development of NSCLC. 
Table 3

Expression of five differential genes in three disease sample groups (mean \pm SD)

\begin{tabular}{|c|c|c|c|c|c|}
\hline Indicators & $\begin{array}{l}\text { COPD group } \\
\text { ( } n=24 \text { cases) }\end{array}$ & $\begin{array}{l}\text { NSCLC group } \\
\text { ( } n=26 \text { cases) }\end{array}$ & $\begin{array}{l}\text { Complication group } \\
\text { ( } n=4 \text { cases) }\end{array}$ & $F$ & $P$ \\
\hline ITGB1 & $1.036 \pm 0.299$ & $5.348 \pm 1.974 \triangle$ & $6.314 \pm 1.485^{\triangle}$ & 40.061 & 0.000 \\
\hline VIM & $1.028 \pm 0.252$ & $1.403 \pm 0.563 \triangle$ & $2.045 \pm 0.222 \triangle \Delta$ & 11.379 & 0.000 \\
\hline KRT14 & $1.033 \pm 0.283$ & $0.937 \pm 0.236$ & $0.668 \pm 0.086 \triangle$ & 3.769 & 0.030 \\
\hline MMP-9 & $1.012 \pm 0.152$ & $1.438 \pm 0.734 \triangle$ & $5.057 \pm 0.541 \triangle \Delta$ & 14.970 & 0.001 \\
\hline Notch1 & $1.008 \pm 0.130$ & $1.086 \pm 0.197$ & $1.224 \pm 0.152^{\triangle}$ & 3.389 & 0.041 \\
\hline
\end{tabular}

Expression of EMT-related genes in the lung adenocarcinoma and the lung squamous cell

The results of this study suggested that only MMP-9 and Notch1 were differentially expressed in two disease sample groups of lung adenocarcinoma and squamous cell lung carcinoma (Table 4 and Figure. 5). MMP-9 and Notch1 were highly expressed in the lung adenocarcinoma group compared with the lung squamous cell carcinoma group $(P<0.05)$, suggesting that MMP-9 and Notch1could be related to the occurrence and development of lung adenocarcinoma.

Table 4

Expression of five differential genes in lung adenocarcinoma and squamous cell lung carcinoma (mean \pm SD)

\begin{tabular}{|lllll|}
\hline Indicators & $\begin{array}{l}\text { Lung adenocarcinoma } \\
\text { group } \\
(\mathbf{n}=\mathbf{1 5} \text { cases })\end{array}$ & $\begin{array}{l}\text { Squamous cell lung carcinoma } \\
\text { group } \\
(\mathbf{n}=\mathbf{1 1} \text { cases })\end{array}$ & $\boldsymbol{t}$ & $\boldsymbol{P}$ \\
\hline ITGB1 & $5.55 \pm 2.39$ & $5.08 \pm 1.26$ & 0.595 & 0.557 \\
\hline VIM & $1.39 \pm 0.64$ & $1.42 \pm 0.46$ & -0.129 & 0.898 \\
\hline KRT14 & $0.93 \pm 0.22$ & $0.94 \pm 0.27$ & -0.075 & 0.941 \\
\hline MMP-9 & $1.67 \pm 0.89$ & $1.12 \pm 0.17$ & 2.354 & 0.032 \\
\hline Notch1 & $1.15 \pm 0.21$ & $0.99 \pm 0.14$ & 2.234 & 0.035 \\
\hline
\end{tabular}


EMT-related genes regulate the fundamental underlying pathogenic process in airways, which is of vital importance in the occurrence and development of NSCLC and COPD ${ }^{[17,18]}$. In this study, we examined the differencein expression of EMT-related genes between COPD and NSCLC. Five candidate differential EMTrelated genes (ITGB1, VIM, KRT14, MMP-9, and Notch1) were found between COPD and NSCLC.The expressions of ITGB1, VIM, and MMP-9 were higher in the NSCLC group compared to the COPD group.Moreover, the expressions of ITGB1, VIM, MMP-9, and Notch1 were higher, and the expression of KRT14 was lower in the complicated group compared to the NSCLC group $(P<0.05)$, which suggests that these genes might be closely related to the occurrence and development of NSCLC, mainly lung adenocarcinoma.

ITGB1 is a cell adhesion protein mainly found in epithelial cells. It participates in cells' survival, motion, assembly of extracellular matrix, adhesion of cells to cell-matrix, immune defense response, and transduction pathways of related signals in order to maintain physiological stability ${ }^{[19]}$. Abnormal expression of ITGB1 activates growth receptors and downstream cell signals to induce EMT, thus promoting abnormal cell proliferation and malignant transformation. Downregulation of ITGB1 can suppress EMT and halt the proliferation and metastasis of tumor cells ${ }^{[20]}$. InNSCLC, ITGB1 promotes the metastasis and invasion of cells by adjusting EMT. With the abnormal expression of ITGB1 in tracheal epithelial cells, EMT is regulated by the signal pathway of mediated mitogen-activated protein kinase (MAPK), which promotes excessive proliferation of airway epithelium, airway fibrosis, and airway remodeling, leading to COPD ${ }^{[21]}$. Plosaet $a^{[22]}$ found that the silencing of ITGB1 can cause inflammation of the airways and lungs and lead to airway remodeling and emphysema, thus inducing COPD, which is thought to be related to the occurrence of EMT.

VIM is a human intermediate filament protein (IF). Under normal physiological conditions, VIM is widely expressed in mesenchymal cells,where it participates in the plasticity to maintain normal cell structure and resist the effect of stress injury ${ }^{[23]}$. Upregulation of VIM in lung epithelial cells induces EMT, leading to the occurrence, metastasis, and invasion of NSCLC. Moreover, Ye et $a^{[25]}$ found that the upregulation of VIM expression in NSCLC tissues promotes metastasis of tumor lymph node and further affects staging and prognosis of the tumor, while its suppression can reduce this process. VIM is highly expressed in airway epithelium and is involved in airway fibrosis and remodeling ${ }^{[26]}$. Mahmood et al[27] found that VIM expression was significantly increased in small airways where it induced EMT to cause small airway fibrosis, leading to COPD.

MMP-9 is a common MMP. When coupled with TIMPs, it regulates elastin and collagen homeostasis and maintains the respiratory tract's structural integrity. The upregulation of MMP-9 mediated by FOXP3 promotes the signal pathway of wnt-B-catenin, induces EMT, and promotes tumor growth and metastasis of NSCLC. ${ }^{[28,30]}$ Dong et a ${ }^{[29]}$ suggested that the suppression of MMP-9 expression promotes EMT and further inhibits metastasis and invasion of NSCLC. Moreover, studies have found that cigarette smoking (CS), dust, and other hazardous substances induce EMT by enabling alveolar macrophages to produce excessive protease, including MMP-9, which leads to alveolar damage and epithelial fibrosis and may, in 
turn, lead to COPD. Aad et a ${ }^{[31]}$ determined that MMP-9 could be used as a biomarker for the staging and severity of COPD by detecting the level of MMP-9 mRNA in peripheral blood samples of COPD patients. Furthermore, Wells and colleagues ${ }^{[32]}$ found that the increase of MMP-9 is correlated with the risk of disease progression of COPD; thus, it can serve as a prognostic biomarker and potential therapeutic target for COPD. Also, Zhou et al[33] demonstrated that CS induces high expression of MMP-9 in alveolar macrophages, causing alveolar damage, which can result in emphysema and COPD.

Notch is a highly conservative signal transduction pathway involved in cell differentiation ${ }^{[34]}$. Notch1 can induce EMT by activating the signal pathway. In addition, Notch1 is associated with tumorigenesis, metastasis, and drug resistance. The overexpression of Notch1 mediates Notch's signal transduction to induce EMT, which not only activates cell proliferation and antagonizes cell death but also participates in the invasion and metastasis of lung cancer. Yuan et $a^{\left[{ }^{[35]}\right.}$ conducted ameta-analysis and found that Notch (mainly Notch1 and Notch3) is a valuable biomarker for predicting the progression of NSCLC. They also reported that a high expression of Notch was positively correlated with lymph node metastasis. However, Gao et $a^{[36]}$ found that the expression of Notch1 in the tissues of NSCLC patients could also inhibit the proliferation and migration of tumor cells. Moreover, a higher expression level of activated Notch1 and HEY2 genewere found in COPD patients. The high expression of Notch1 activates the signal transduction pathway and induces EMT, which can significantly promote the development of airway myofibroblasts and further cause tracheal fibrosis, resulting in COPD. In addition, the signal transduction of Notch can prevent the production of reactive oxygen species (ROS), inhibit the apoptosis of tracheal epithelial cells, cause airway lesions, and accelerate the further development of COPD

KRT14,a keratin used to maintain the normal shape and function of epithelial cells, is expressed in airway fibrosis and alveolar damage,whichcan repair injured epithelial cells ${ }^{[38]}$. Arumugam et a ${ }^{[39]}{ }_{\text {reported that a }}$ continuous expression of KRT14 led to multiple tumors in mice, suggesting that the high expression of KRT14 could induce tumors. Ooi et $a^{[40]}$ found that KRT14 can promote metastasis of NSCLC. They alsorevealed thatcontinuous expression of KRT14 in airway epithelial cells often suggests an unfavorable prognosis. KRT14 is an important marker for basal cells of the trachea and bronchus,affecting the differentiation of basal cells and specifically changing the cell composition of airway epithelium. In addition, KRT14 participates in EMT, thereby causing trachea remodeling and leading to COPD ${ }^{[41]}$. Schamberger et $a^{[42]}$ found that the specific expression increase of KRT14 in the bronchial epithelium of smokers and COPD patients results in lung tissue damage, especially alveolar damage, and a higher risk of disease.

There might be some difference between previous studies reporting on ITGB1, VIM, KRT14, MMP-9, Notch1, and our results: 1) this study had a relatively small sample size, which may lead to bias. 2) Smoking is an important risk factor of lung cancer and COPD; Yet, in this study, we did not compare smokers with non-smokers and conduct stratified comparison and analysis, which might have implications for the final results. 3) Most of the patients studied live in the plateau region; a high-altitude environment with scarce oxygen might affect the results. 4) Due to multiple signal pathways in EMT, 
there might be differences in signal pathways induced by EMT-related genes. 5) Different conditions for experiments could lead to slight discrepancies in the results. 6) In this study, no analysis has been conducted on the potential implications of complications as diabetes, hypertension, and other tumors on the results.

So far, only a few studies have investigated the difference in EMT-related genes among various pathological types of NSCLC. This study first reported the difference in EMT-related genes between lung adenocarcinoma and lung squamous cell carcinoma. Our data suggested that MMP-9 and Notch1 were more highly expressed in the lung adenocarcinoma group than in the squamous cell lung carcinoma group. Still, further in-depth verification and research are required to confirm these findings.

\section{Conclusions}

Our results show differences suggesting that here is greater EMT activity in the cancer versus COPD group and even more in the combined group. And our results also show differences between adeno- and squamous cell cancer types though numbers here seem very small. Despite the above-listed limitations, the study has managed to roughly demonstrate the correlation of EMT in NSCLC and COPD incidence. These data may help differentiate NSCLC patients from those with COPD. Yet, a future multicenter study with larger sample size is required to furtherconfirm these findings further.In addition, a comparison between smokers and non-smokers should be conducted coupled with stratified comparative studies to obtain more statistical data. Meanwhile, studies on the implications of EMT-related genes on the survival time, staging, efficacy evaluation, disease prediction, and prognosis of NSCLC patients could also substantially contribute to this research issue.

\section{Abbreviations}

EMT: Epithelial-mesenchymal transformation; NSCLC: Non-small cell lung cancer; COPD: Chronic obstructive pulmonary disease; WHO: World health organization; ITGB1: Integrin beta 1; KRT14: Keratin 14; MMP-9: Matrix metallo protein 9; Notch1: Notches1; VIM: Vimentin; KEGG: Kyoto encyclopedia of genes and genomes; NCBI: National center for biotechnology information; MAPK: Mitogen-activated protein kinase; IF: Intermediate filament protein; CS: Cigarette smoking; ROS: Reactive oxygen species; ERK: Extracellular regulated protein kinases.

\section{Declarations}

\section{Ethics approval and consent to participate}

The study was carried out according to the principles of the Declaration of Helsinki and the prevailing norms for performing investigation in humans. Data confidentiality was ensured according to the Law of Data Protection. The study was approved by the Ethical Committee and Clinical Investigation of Qinghai 
University Affiliated Hospital and The Third People's Hospital of Yuhang District, and all the participants provided written informed consent.

\section{Consent for publication}

Not applicable.

\section{Availability of data and materials}

Data are available from the authors under request.

\section{Competing interests}

The authors declare that they have no competing interests.

\section{Funding}

This study was supported by funding from Basic Research Program of Qinghai Provincial Department of Science and Technology of China (2017-ZJ-710) and The Science and technology Project of Hangzhou of Zhejiang Province of China (B20210376).

\section{Authors' contributions}

The study concept and design: Jun Jiang. Collected samples of patients: Lian-Gang Li, Hangqierang Cai, Xiao-Bo Zhou, Lan Zhang, Zhi-Bo Liu, Tai Jiu, Fang Hui, Ya-Jun Tuo. The acquisition of the data: LianGang Li, Hangqierang Cai. The statistical analysis: Lian-Gang Li, Jun Jiang. The drafting of the manuscript: Lian-Gang Li. Critical revision and approval for submission: Lian-Gang Li, Hangqierang Cai, Xiao-Bo Zhou, Lan Zhang, Zhi-Bo Liu, Tai Jiu, Fang Hui, Ya-Jun Tuo, Jun Jiang. All authors read and approved the final manuscript.

\section{Acknowledgments}

Jun Jiang is the recipient of Basic Research Program of Qinghai Provincial Department of Science and Technology of China (2017-ZJ-710). Lian-Gang Li is the recipient of The Science and technology Project of Hangzhou of Zhejiang Province of China(B20210376). The authors thank Tai Jiu, Ya-Jun Tuo and Fang Hui for providing samples of patients and valuable suggestions and technical assistance.

\section{Authors' information}

1Department of Oncology, The Third People's Hospital of Yuhang District, No. 8, Yangshanwan Road, Pingyao Town, Yuhang District, Hangzhou, Zhejiang 311115, People's Republic of China. ${ }^{2}$ Department of Oncology, Qinghai University Affiliated Hospital, No. 29, Tongren Road, Chengxi District, Xining, Qinghai 810001, People's Republic of China. ${ }^{3}$ Department of Respiratory, Qinghai Provincial People's Hospital, No. 2, Gonghe Road, Chengdong District, Xining, Qinghai 810007, People's Republic of China. 


\section{References}

[1] Barnett R. Lung cancer [J]. Lancet (London, England), 2017, 390(10098): 928.

[2] Nasim F, Sabath B F, Eapen G A. Lung Cancer [J]. Med Clin North Am, 2019, 103(3): 463-73.

[3] O'leary K, Shia A, Schmid P. Epigenetic Regulation of EMT in Non-Small Cell Lung Cancer [J]. Curr Cancer Drug Targets, 2018, 18(1): 89-96.

[4] Rabe K F, Watz H. Chronic obstructive pulmonary disease [J]. Lancet, 2017, 389(10082): 1931-40.

[5] Brown P J. The ravages of COPD [J]. The Lancet Respiratory medicine, 2018, 6(5): 336.

[6] Celli B R, Wedzicha J A. Update on Clinical Aspects of COPD. Reply [J]. The New England journal of medicine, 2019, 381(25): 2485-6.

[7] Zhong N S, Zeng G Q. Prevention and treatment of chronic respiratory diseases in China [J]. Chronic diseases and translational medicine, 2019, 5(4): 209-13.

[8] 2019 exceptional surveillance of lung cancer: diagnosis and management (NICE guideline NG122). London; National Institute for Health and Care Excellence (UK). 2019.

[9] Biswas A, Mehtaeh H J, FOLCH E E. Chronic obstructive pulmonary disease and lung cancer: interrelationships [J]. Current opinion in pulmonary medicine, 2018, 24(2): 152-60.

[10] Jolly M K, Ward C, Eapen M S, et al. Epithelial-mesenchymal transition, a spectrum of states: Role in lung development, homeostasis, and disease [J]. Developmental dynamics : an official publication of the American Association of Anatomists, 2018, 247(3): 346-58.

[11] Sulaiman A, Yao Z M, Wang L S. Re-evaluating the role of epithelial-mesenchymal-transition in cancer progression [J]. Journal of biomedical research, 2018, 32(2): 81-90.

[12] Lamouille S, Xu J, Derynck R. Molecular mechanisms of epithelial-mesenchymal transition [J]. Nature reviews Molecular cell biology, 2014, 15(3): 178-96.

[13] Skrypek N, Goossans S, De Smedt E, et al. Epithelial-to-Mesenchymal Transition: Epigenetic Reprogramming Driving Cellular Plasticity [J]. Trends Genet, 2017, 33(12): 943-59.

[14] Sohal S S. Airway Basal Cell Reprogramming and Epithelial-Mesenchymal Transition: A Potential Key to Understanding Early Chronic Obstructive Pulmonary Disease [J]. Am J Respir Crit Care Med, 2018, 197(12): 1644-5.

[15] Eapen M S, Myers S, Lu W, et al. SE-cadherin and sVE-cadherin indicate active epithelial/endothelial to mesenchymal transition (EMT and EndoMT) in smokers and COPD: implications for new biomarkers 
and therapeutics [J]. Biomarkers : biochemical indicators of exposure, response, and susceptibility to chemicals, 2018, 23(7): 709-11.

[16] Eapen M S, Sharma P, Thompson I E, et al. Heparin-binding epidermal growth factor (HB-EGF) drives EMT in patients with COPD: implications for disease pathogenesis and novel therapies [J]. Lab Invest, 2019, 99(2): 150-7.

[17] Mahmood M Q, Ward C, Muller H K, et al. Epithelial mesenchymal transition (EMT) and non-small cell lung cancer (NSCLC): a mutual association with airway disease [J]. Medical oncology (Northwood, London, England), 2017, 34(3): 45.

[18] Hou W, Hu S, Li C, et al. Cigarette Smoke Induced Lung Barrier Dysfunction, EMT, and Tissue Remodeling: A Possible Link between COPD and Lung Cancer [J]. BioMed research international, 2019, $2019(2025636$.

[19] Zheng W, Jiang C, Li R. Integrin and gene network analysis reveals that ITGA5 and ITGB1 are prognostic in non-small-cell lung cancer [J]. Onco Targets Ther, 2016, 9(8): 2317-20.

[20] Ding Y, Pan Y, Liu S, et al. Elevation of MiR-9-3p suppresses the epithelial-mesenchymal transition of nasopharyngeal carcinoma cells via down-regulating FN1, ITGB1 and ITGAV [J]. Cancer Biol Ther, 2017, 18(6): 414-24.

[21] Wright D B, Meurs H, Dekkers B G J. Integrins: therapeutic targets in airway hyperresponsiveness and remodelling? [J]. Trends Pharmacol Sci, 2014, 35(11): 567-74.

[22] Plosa $E \mathrm{~J}$, Benjamin $\mathrm{J} T$, Sucre $\mathrm{J}$ M, et al. $\beta 1$ Integrin regulates adult lung alveolar epithelial cell inflammation [J]. JCl Insight, 2020, 5(2): 129259.

[23] Battaglia R A, Delic S, Herrmann $\mathrm{H}$, et al. Vimentin on the move: new developments in cell migration [J]. F1000Res, 2018, 7(9): 1794-6.

[24] Bronte G, Puccetti M, Crinò L, et al. Epithelial-to-mesenchymal transition and EGFR status in NSCLC: the role of vimentin expression [J]. Ann Oncol, 2019, 30(2): 339-40.

[25] Ye Z, Zhang X, Luo Y, et al. Prognostic Values of Vimentin Expression and Its Clinicopathological Significance in Non-Small Cell Lung Cancer: A Meta-Analysis of Observational Studies with 4118 Cases [J]. PLoS One, 2016, 11(9): 163162-5.

[26] Li F J, Surolia R, Li H, et al. Low-dose cadmium exposure induces peribronchiolar fibrosis through site-specific phosphorylation of vimentin [J]. Am J Physiol Lung Cell Mol Physiol, 2017, 313(1): L80-L91.

[27] Mahmood M Q, Sohal S S, Shukla S D, et al. Epithelial mesenchymal transition in smokers: large versus small airways and relation to airflow obstruction [J]. International journal of chronic obstructive pulmonary disease, 2015, 10(7): 1515-24. 
[28] Yang S, Liu Y, Li M-Y, et al. FOXP3 promotes tumor growth and metastasis by activating Wnt/ $\beta$ catenin signaling pathway and EMT in non-small cell lung cancer [J]. Mol Cancer, 2017, 16(1): 124-7.

[29] Dong Y Z, Meng X M, Li G S. Long non-coding RNA SNHG15 indicates poor prognosis of non-small cell lung cancer and promotes cell proliferation and invasion [J]. Eur Rev Med Pharmacol Sci, 2018, 22(9): 2671-9.

[30] Li D, Xu T, Wang X, et al. The role of ATP8A1 in non-small cell lung cancer [J]. Int J Clin Exp Pathol, 2017, 10(7): 7760-6.

[31] Abd El-Fatah M F, Ghazy M A, Mostafa M S, et al. Identification of MMP-9 as a biomarker for detecting progression of chronic obstructive pulmonary disease [J]. Biochem Cell Biol, 2015, 93(6): 541-7.

[32] Wells J M, Parker M M, Oster R A, et al. Elevated circulating MMP-9 is linked to increased COPD exacerbation risk in SPIROMICS and COPDGene [J]. JCI Insight, 2018, 3(22): 123614.

[33] Zhou L, Le Y, Tian J, et al. Cigarette smoke-induced RANKL expression enhances MMP-9 production by alveolar macrophages [J]. International journal of chronic obstructive pulmonary disease, 2018, 14(6): 81-91.

[34] Zong D, Ouyang R, Li J, et al. Notch signaling in lung diseases: focus on Notch1 and Notch3 [J]. Ther Adv Respir Dis, 2016, 10(5): 468-84.

[35] Yuan X, Wu H, Xu H, et al. Meta-analysis reveals the correlation of Notch signaling with non-small cell lung cancer progression and prognosis [J]. Scientific reports, 2015, 5(3): 10338-40.

[36] Gao Y P, Li Y, Li H J, et al. LncRNA NBR2 inhibits EMT progression by regulating Notch1 pathway in NSCLC [J]. Eur Rev Med Pharmacol Sci, 2019, 23(18): 7950-8.

[37] Zong D, Li J, Cai S, et al. Notch1 regulates endothelial apoptosis via the ERK pathway in chronic obstructive pulmonary disease [J]. Am J Physiol Cell Physiol, 2018, 315(3): C330-C40.

[38] Paraskevopoulou V, Papafotiou G, Klinakis A. KRT14 marks bladder progenitors [J]. Cell Cycle, 2016, 15(23): 3161-2.

[39] Arumugam A, Weng Z, Chaudhary S C, et al. Keratin-6 driven ODC expression to hair follicle keratinocytes enhances stemness and tumorigenesis by negatively regulating Notch [J]. Biochemical and biophysical research communications, 2014, 451(3): 394-401.

[40] Ooi A T, Mah V, Nickerson D W, et al. Presence of a putative tumor-initiating progenitor cell population predicts poor prognosis in smokers with non-small cell lung cancer [J]. Cancer research, 2010, 70(16): 6639-48. 
[41] Milara J, Peiró T, Serrano A, et al. Epithelial to mesenchymal transition is increased in patients with COPD and induced by cigarette smoke [J]. 2013, 68(5): 410-20.

[42] Schamberger A C, Staab-Weijnitz C A, Mise-Racek N, et al. Cigarette smoke alters primary human bronchial epithelial cell differentiation at the air-liquid interface [J]. Scientific reports, 2015, 5(8): 8163-6.

\section{Figures}

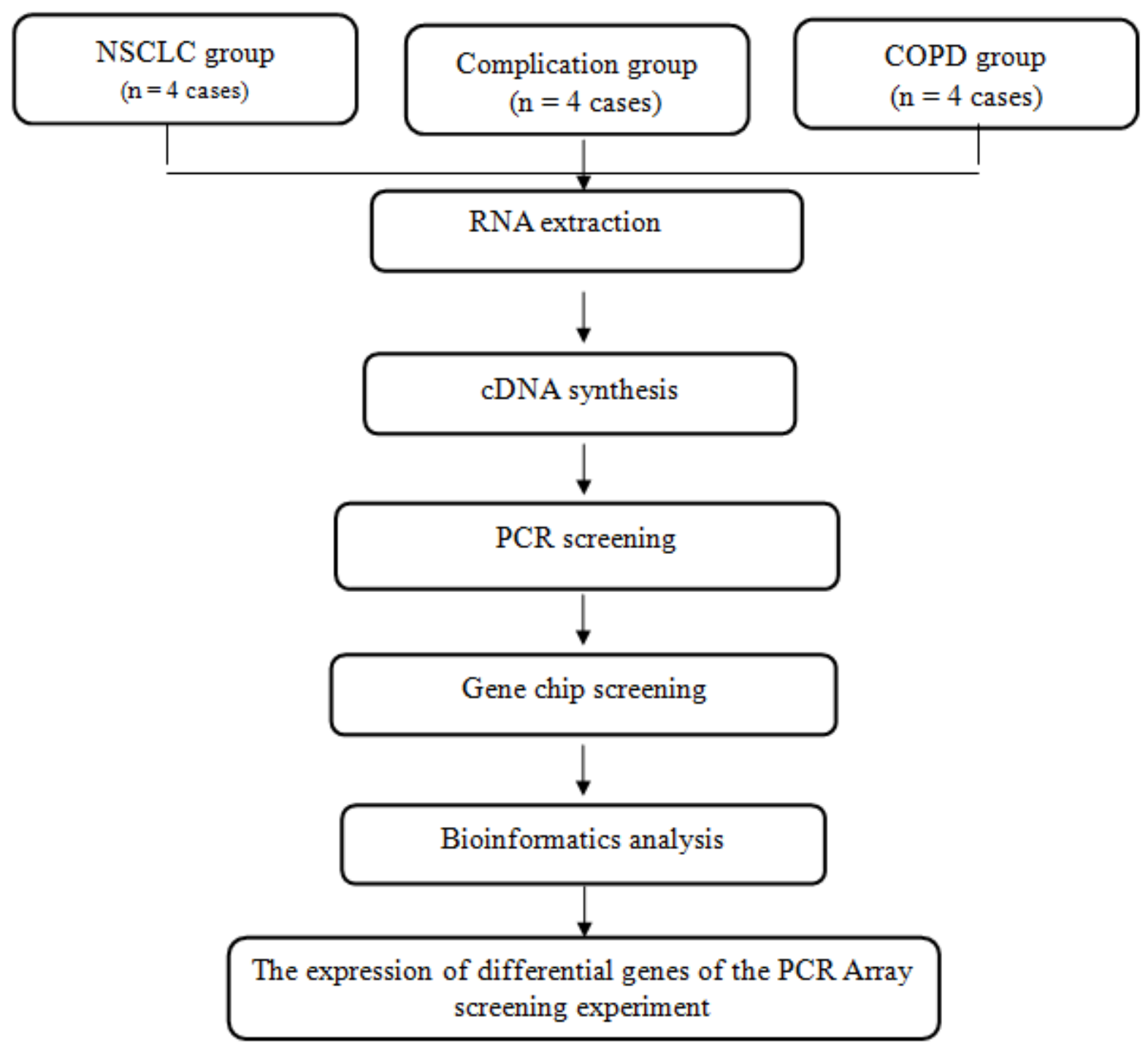

\section{Figure 1}

The PCR Array screening experiment 


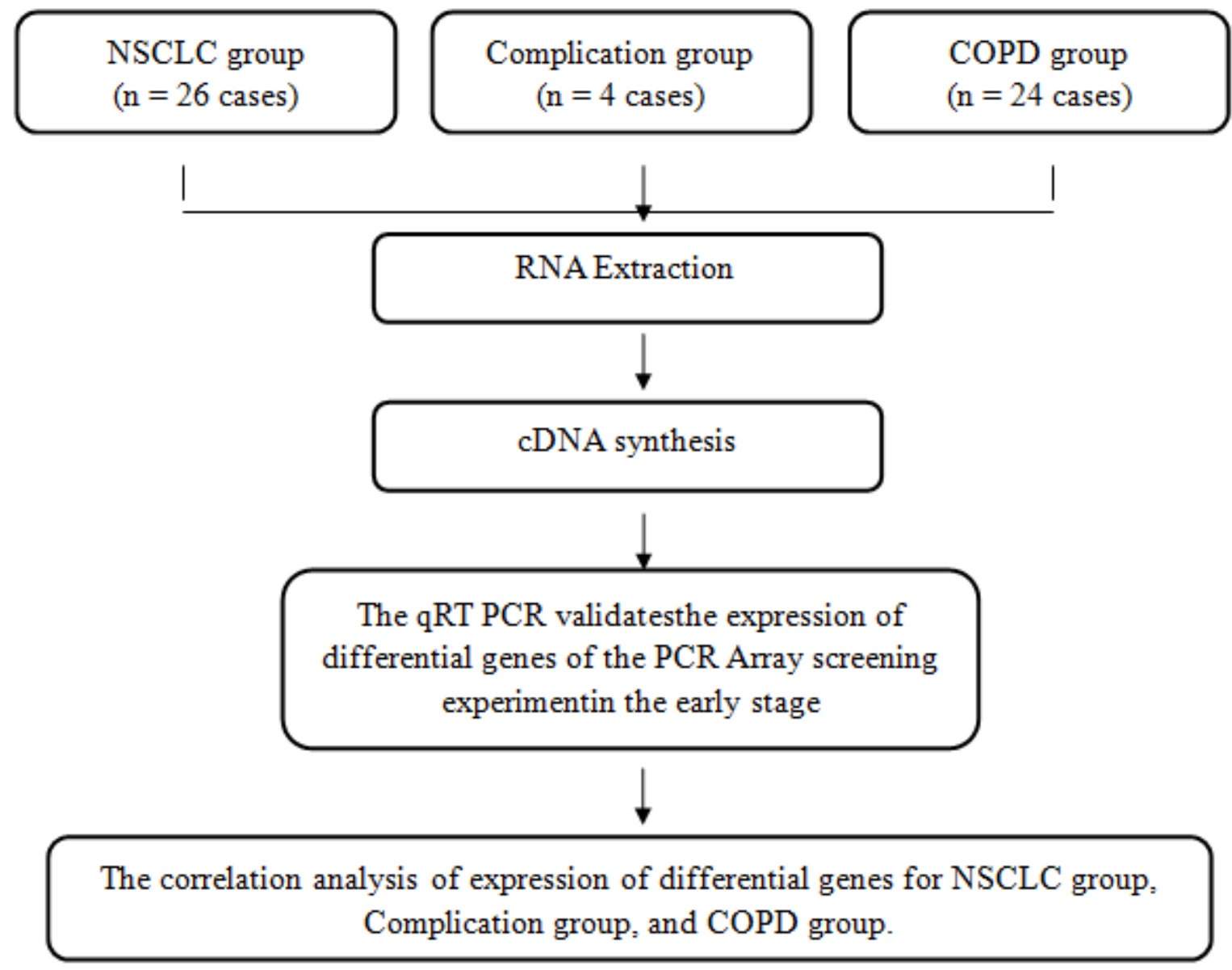

Figure 2

The qRT PCR Array screening experiment

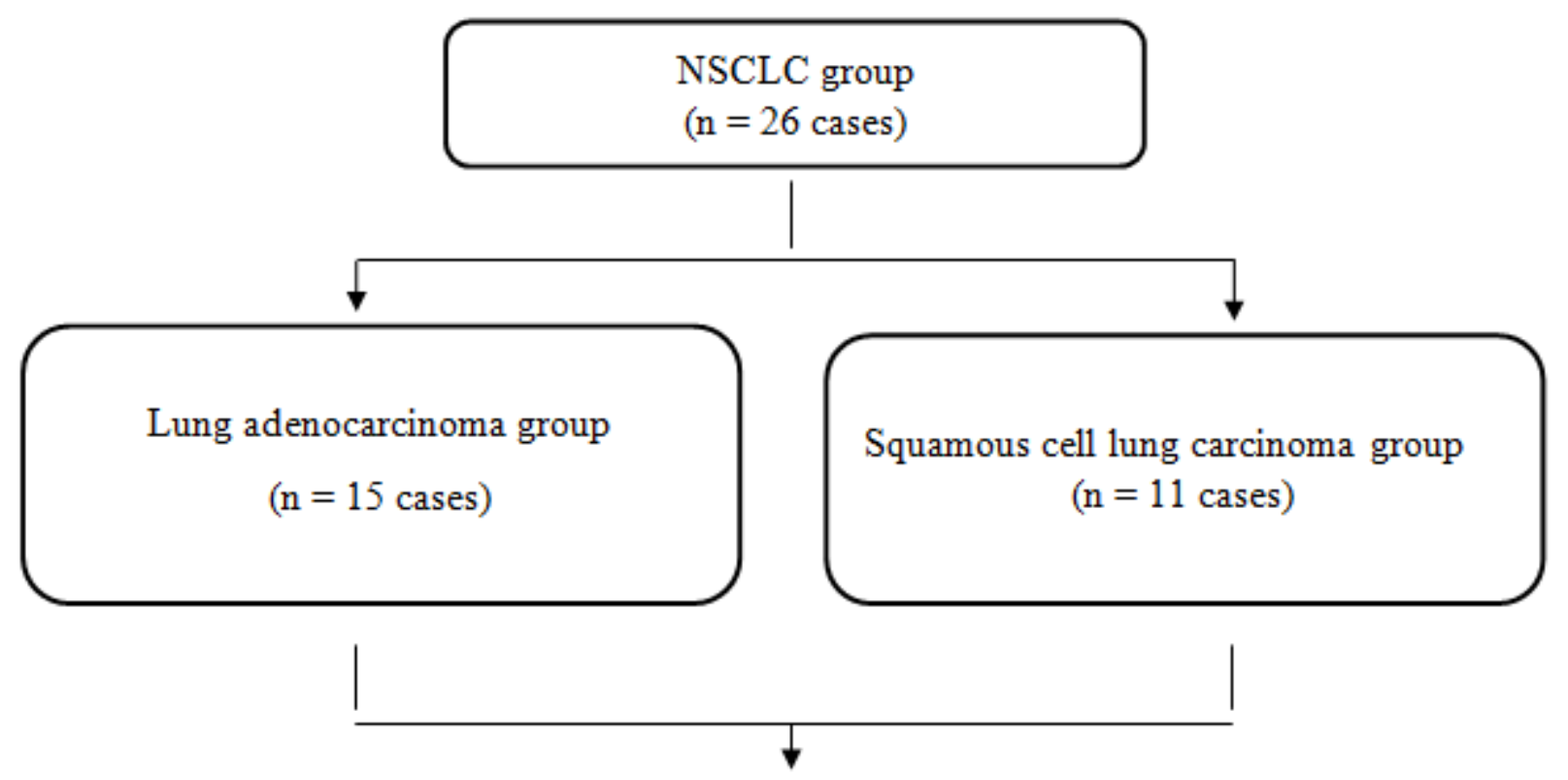

The correlation analysis of expression of differential genes for Lung adenocarcinoma group and Squamous cell lung carcinoma group 
Figure 3

Expression of five differential genes in lung adenocarcinoma and squamous cell lung carcinoma flowchart
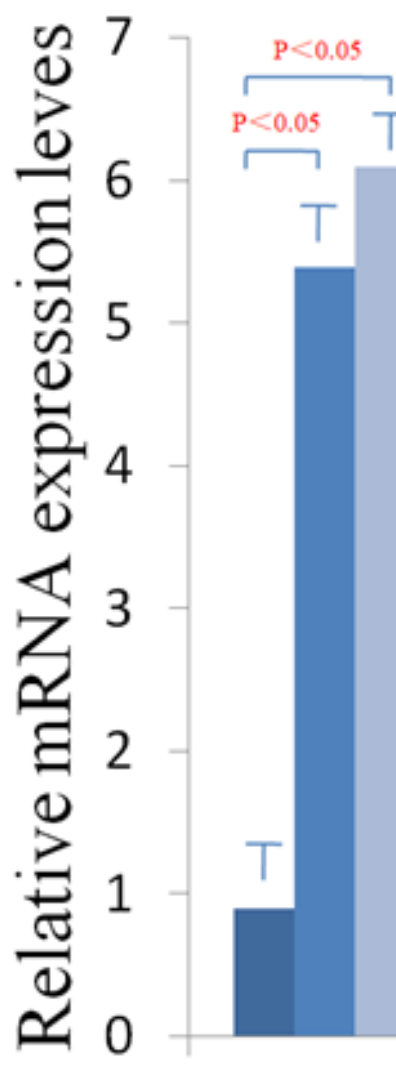

- COPD group

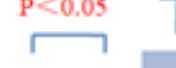
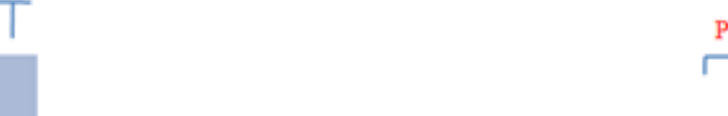

$\mathrm{P}<0.05$

- NSCLC group

Complication group

\section{Figure 4}

Expression maps of five differential genes in the cell precipitate of bronchoalveolar lavage fluid from COPD, NSCLC, and Complication groups 


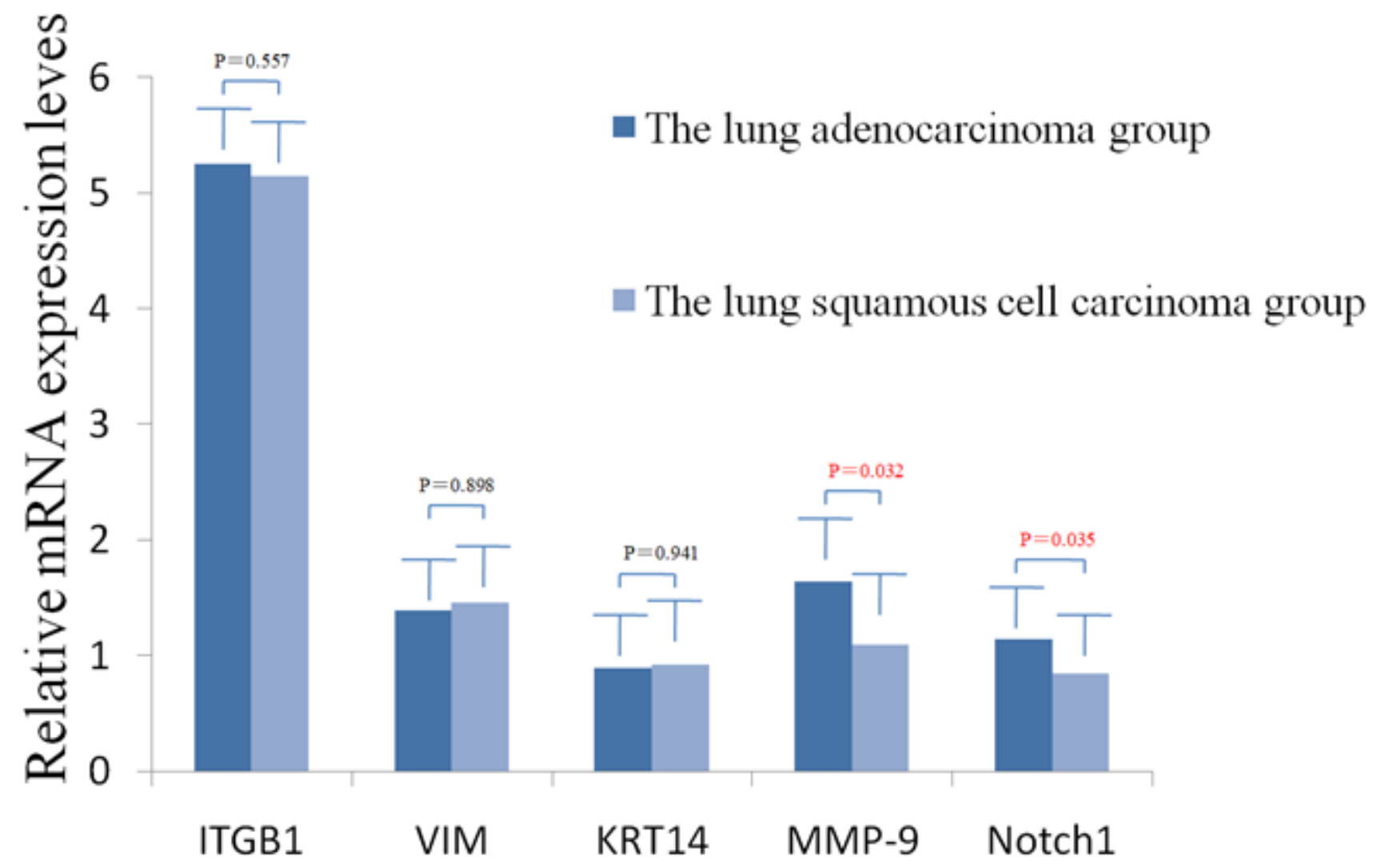

Figure 5

Expression maps of 5 differential genes in the cell precipitate of bronchoalveolar lavage fluid of lung adenocarcinoma and squamous cell lung carcinoma.

\section{Supplementary Files}

This is a list of supplementary files associated with this preprint. Click to download.

- Supplementaryfigures.docx

- Supplementarytables.docx 